Methods From January 2010 to November 2012, all females and MSM were routinely tested for anorectal, oropharyngeal and urogenital Chlamydia trachomatis $(\mathrm{Ct})$ and Neisseria gonorrhoeae $(\mathrm{Ng})$. Data were collected on demographics and sexual behaviour.

Results The study population comprised 4829 consults. Overall Ct positivity was $6.8 \%(131 / 1937)$ in females and $10.0 \%(289 / 2892)$ in MSM, for $\mathrm{Ng}$ this was $4.3 \%(\mathrm{n}=83)$ and $6.7 \%(\mathrm{n}=193)$ respectively. The proportion isolated anorectal Ct was $16.8 \%(\mathrm{n}=22)$ in females and $63.0 \%(\mathrm{n}=182)$ in MSM, for $\mathrm{Ng}$ this was $10.8 \%(\mathrm{n}=9)$ and $44.0 \%(n=85)$ respectively. Combined urogenital and anorectal $\mathrm{Ct}$ was $45.0 \%(n=59)$ in females and $13.5 \%(n=39)$ in MSM, for $\mathrm{Ng}$ this was $19.3 \%(n=16)$ and $11.4 \%(n=22)$ respectively. Isolated oropharyngeal Ct was $9.9 \%(n=13)$ in females and $5.9 \%(n=17)$ in MSM, for $\mathrm{Ng}$ this was $53.0 \%(\mathrm{n}=44)$ and $31.6 \%(\mathrm{n}=61)$ respectively. Isolated urogenital Ct was $28.2 \%(n=37)$ in females and $17.6 \%(n=51)$ in MSM, for $\mathrm{Ng}$ this was $16.9 \%(\mathrm{n}=14)$ and $13.0 \%$ $(\mathrm{n}=25)$ respectively.

Discussion Anorectal and oropharyngeal STD were often isolated and thereby not coincidentally treated with urogenital STD. Therefore urogenital testing only is insufficient. Furthermore, these isolated infections remain undiagnosed if routine anorectal/ oropharyngeal testing is lacking. Combined anorectal/urogenital $\mathrm{Ct} / \mathrm{Ng}$ were also highly prevalent, highlighting the importance of an effective treatment for simultaneous genital and extragenital $\mathrm{Ct} / \mathrm{Ng}$.

\section{P3.155 ASSESSMENT OF KNOWLEDGE OF SEXUAL TRANSMITTED DISEASES (STDS) AND A SEROPREVALENCE STUDY IN GAY MAN IN KAYSERI, TURKEY}

doi:10.1136/sextrans-2013-051184.0613

'E Balci, ${ }^{2} \mathrm{M}$ Doganay, ${ }^{2 \mathrm{H}}$ Demiraslan, ${ }^{3} \mathrm{~S}$ Gokahmetoglu. 'Erciyes University Medical Faculty Department of Public Health, Kayseri, Turkey; ${ }^{2}$ Erciyes University Medical Faculty Department of Infectious Diseases, Kayseri, Turkey; ${ }^{3}$ Erciyes University Medical Faculty Department of Microbiology, Kayseri, Turkey

Background Homosexual behaviour is prone to many sexual transmitted diseases (STDs) and many social problems. The aim of this study is to assess the knowledge, source, the reliability and accuracy of information resources and to assess the seropositivity for some STDs.

Method A questionnaire was prepared for the STDs including these; to learn their demographic and economical characteristics, having any chronic disease or, experienced any disease, use of any illegal drug, their sense for acceptability in public, number of partner in one year, main transmission route, prevention and source of their information about this diseases in gay people. In additional these, Another questions were to learn their to join an education programme for prevention of STDs and to give blood samples for the serologic investigation against hepatitis B, hepatitis C, syphilis and HIV. Voluntary people defined himself as gay, was entered to the study.

Results The questionnaire was replied by 94 people. Mean age was $23.32 \pm 4.6$ year and $80.4 \%$ defined himself as gay. Of these, $72.6 \%$ was graduated from high school or university, $5.9 \%$ had a chronic disease and 3.9\% had receiving any medicine. $29.4 \%$ had no any health insurance. Using one of these; cigarette, alcohol, heroin or cocaine were at percentage of $62.8,46.8,10.6$ and1.1 respectively. $23.4 \%$ had multiple partners and $76.7 \%$ single partners. Majority people knew the transmission of HIV/AIDS by sexual intercourse (88.3\%) or blood/blood products (77.7\%). $69.1 \%$ had a history of unprotected sexual relation. Major source of their knowledge about STDs was internet or other media. Blood samples could be taken from 39, one was positive for HIV. An education programme was used as a small group after questionnaire.

Conclusion This is a first basic study on homosexual group, summarising their problems in our region.

\section{P3.156 CONTRIBUTION OF SEXUAL PRACTISES OTHER THAN ANAL SEX TO THE TRANSMISSION OF BACTERIAL SEXUALLY TRANSMITTED INFECTIONS IN MEN WHO HAVE SEX WITH MEN}

doi:10.1136/sextrans-2013-051184.0614

1,2,3 L Nash, 1,2 J S Hocking, 1,2T R H Read, 1,2M Y Chen, 1,2,3 C S Bradshaw, 1,2D S Forcey, ${ }^{1,2} \mathrm{C}$ K Fairley. 'Melbourne Sexual Health Centre, Melbourne, Australia; ${ }^{2}$ Sexual Health Unit, School of Population Health, University of Melbourne, Melbourne, Australia; ${ }^{3}$ Monash University, Melbourne, Australia

Background Syphilis, chlamydia and gonorrhoea are increasingly common amongst Australian men who have sex with men (MSM). Our objective was to quantify the proportion of cases and risk of primary syphilis (PS), urethral chlamydia (UCT) and urethral gonorrhoea (UGC) attributable to sexual practises other than anal sex. Methods Electronic medical records for MSM who attended Melbourne Sexual Health Centre between July 2002 (for PS) or January 2006 (for UCT and UGC) and October 2012 inclusive were examined.

Results There were 37533 eligible consultations; 2374 (6\%) of these reported no anal sex. There were 204 PS diagnoses, 673 UCT diagnoses, and 618 UGC diagnoses; 12 (6\%), 16 (2\%) and 44 (7\%) cases respectively occurred in consultations where no anal sex was reported in the previous three months (PS, UGC) or twelve months (UCT). Amongst MSM who reported no anal sex, PS was diagnosed in 0.5 cases $/ 100$ consultations, UCT was diagnosed in 1.5 cases $/ 100$ tests for UCT and UGC was diagnosed in 14 cases/100 tests for UGC. UCT was significantly more common in MSM who reported anal sex (OR 2.18, 95\% CI $1.32-3.59, p=0.002)$, but PS ( $p=0.82$ ) and UGC ( $p=0.14)$ were not. For MSM who reported anal sex, condom use was protective for all three infections (all $p \leq 0.03$ ).

Conclusions UCT rarely occurs in MSM due to sexual practises other than anal sex; however these practises appear to contribute significantly to the acquisition of PS and UGC. Our figures probably underestimate the risk involved, as men who have anal sex also engage in other sexual practises. The implication is that successful programmes to control sexually transmitted infections, particularly PS and UGC, will need to utilise additional strategies, such as frequent testing, as well as promoting condom use.

\section{P3.157 INCREASING STI RATES IN YOUNG MSM IN THE NETHERLANDS, 2006-2011}

doi:10.1136/sextrans-2013-051184.0615

${ }^{1}$ F D H Koedijk, 'B H van Benthem, ${ }^{2}$ E M D C Vrolings, ${ }^{1,3} \mathrm{M}$ A B van der Sande, ${ }^{4} \mathrm{On}$ behalf of the Dutch STI clinics. 'National Institute of Public Health and the Environment, Bilthoven, The Netherlands; ${ }^{2}$ STI AIDS The Netherlands, Amsterdam, The Netherlands; ${ }^{3}$ Julius Center for Health Sciences and Primary Care, University Medical Centre Utrecht, Utrecht, The Netherlands; ${ }^{4} A$ van Daal (East), AP van Leeuwen (NorthHolland Flevoland), F de Groot (North), CJPA Hoebe (Limburg), M Langevoort (Utrecht), AM van Camerijk (South-Holland North), J van de Sande (Zeeland-Brabant), V Wieërs (South-Holland South), Bilthoven, The Netherlands

Objective Young men having sex with men (MSM) are a high-risk group involved in on-going transmission of sexually transmitted infections (STI). To improve our understanding of this epidemic, distribution of STI and testing behaviour in young MSM attending STI clinics in the Netherlands was analysed.

Methods National STI surveillance data on young MSM (15-24 years) for 2006-2011 were analysed. STI included were chlamydia, gonorrhoea, infectious syphilis and HIV. Chi-square test for trend was performed to analyse time trends in testing behaviour and STI positivity rates, multivariable logistic regression was used to identify factors associated with STI positivity.

Results Number of consultations in young MSM increased from 1161 in 2006 to 3167 in 2011 (+166\%). The proportion not aware of 